\title{
The Distance to the Large Magellanic Cloud
}

\author{
Piotr Popowski \\ Institute of Geophysics and Planetary Physics, L-413 \\ Lawrence Livermore National Laboratory, University of California \\ P. O. Box 808, Livermore, CA 94551, USA
}

\begin{abstract}
I demonstrate that the two unexpected results in the local Universe: anomalous intrinsic $(V-I)_{0}$ colors of RR Lyrae stars and clump giants in the Galactic center, and very short distances to Magellanic Clouds inferred from clump giants, can be at least partially resolved with a modified coefficient of selective extinction $A_{V} / E(V-I)$. With this modification, I find a new clump-giant distance modulus to the Large Magellanic Cloud, $\mu_{\mathrm{LMC}}=18.27 \pm 0.07$, which is 0.09 larger than the Udalski (1998b) result. When distance estimates from the red clump, RR Lyrae stars and the eclipsing binary HV2274 are combined, one obtains $\mu_{\mathrm{LMC}}=18.31 \pm 0.04$ (internal).
\end{abstract}

\section{Distance to the LMC - Controversy and New Determinations}

The Hubble constant, $H_{0}$, is one of the most important cosmological parameters. There are two major paths to determine $H_{0}$. The more elegant one is non-local and based on observations of the high-redshift Universe. Modeling of gravitational lensing of quasars and observations of the Cosmic Microwave Background both belong to this group of methods. The second path goes through a determination of distances and recession velocities of objects with motions dominated by the Hubble linear expansion. The distances, which are harder to measure than recession velocities, are determined based on a distance-ladder approach. The Magellanic Clouds, and especially the Large Magellanic Cloud (LMC), play a major role in this treatment. Almost all of the extragalactic distance scale is known only relative to the LMC (Madore et al. 1999). Therefore, it is absolutely essential to establish a reliable distance to the LMC ( $\left.d_{\text {LMC }}\right)$. This can be achieved only with an understanding of the systematics inherent in the standard candles used for the distance determinations.

For many years now there has been a division between the so called "short" and "long" distance scales to the LMC. Currently, the measured values of $d_{\text {LMC }}$ span a range of over 25\% (see e.g., Feast \& Catchpole 1997; Stanek, Zaritsky, \& Harris 1998).

Distances are measured based on a general formula

$$
\mu_{\mathrm{LMC}}=X-M_{X}-A_{X}
$$

where $X$ is an apparent magnitude, $M_{X}$ is an absolute magnitude, and $A_{X}$ is an extinction in the band of the observations. The uncertainties in all ingredients 
present in equation (1) may compromise the final answer. Here I am going to concentrate on three new or revised methods that cluster consistently around a short distance to the LMC:

[1] Paczyński \& Stanek (1998) pointed out that clump giants should constitute an accurate distance indicator. Udalski et al. (1998b) and Stanek et al. (1998) applied the clump method and found a very short distance to the LMC ( $\mu_{\mathrm{LMC}} \lesssim 18.2$ ). In response, Cole (1998) and Girardi et al. (1998) suggested that clump giants are not standard candles and that their absolute $I$ magnitudes, $M_{I}(\mathrm{RC})$, depend on the metallicity and age of the population. Udalski (1998a, $1998 \mathrm{~b}$ ) rejected this criticism by showing that the metallicity dependence is at a low level of about $0.1 \mathrm{mag} / \mathrm{dex}$, and that $M_{I}(\mathrm{RC})$ is approximately constant for cluster ages between 2 and $10 \mathrm{Gyr}$. Recent developments (see e.g., Popowski 2000 for a short review) suggest that the absolute character of $M_{I}(\mathrm{RC})$ is a major systematic uncertainty in this method.

[2] Popowski \& Gould (1999) determined the absolute magnitude of RR Lyrae stars, $M_{V}(\mathrm{RR})=0.71 \pm 0.07$ at $[\mathrm{Fe} / \mathrm{H}]=-1.6$, from the statistical parallax, cluster kinematics and trigonometric parallax methods. When this result is coupled with the LMC RR Lyrae photometry of Udalski et al. (1999) and Walker (1992), one obtains $\mu_{\mathrm{LMC}} \approx 18.30 \pm 0.08$. The value of $M_{V}(\mathrm{RR})$ remains the main uncertainty of this determination.

[3] Guinan et a.l. (1998) solved the eclipsing binary HV2274 and obtained various stellar parameters and the distance to the LMC. The spectra used for this purpose did not extend far enough toward long wavelengths, and the $B$ and $V$ photometry was needed to break the degeneracy between the reddening and the shape of the extinction curve. With Udalski et al. (1998c) photometry, Guinan et al. (1998) obtained $\mu_{\mathrm{LMC}}=18.30 \pm 0.07$. Application of Nelson et al. (2000) photometry would result in $\mu_{\mathrm{LMC}}=18.40 \pm 0.07$. The reddening constitutes a major uncertainty.

In two out of three cases the absolute magnitudes of distance indicators are under debate. Due to a huge number of possible environments, it is very hard to prove the standard character of a given candle. However, it should be possible to check whether other stellar characteristics of a candle behave in a predictable fashion. To follow this suggestion, I will concentrate on the stars in the Galactic bulge.

\section{The Mystery of Anomalous Colors in the Galactic Bulge}

Paczyński (1998) tried to explain why the clump giants in the Baade's Window have $(V-I)_{0}$ colors which are approximately 0.2 mag redder than in the solar neighborhood. He could not find any satisfactory answer. Stutz, Popowski, \& Gould (1999) found a corresponding effect for the Baade's Window RR Lyrae stars, which have $(V-I)_{0}$ redder by about 0.17 than their local counterparts. The similar size of the color shift in RR Lyrae stars and clump giants suggests common origin. The bulge RR Lyrae stars and clump giants both burn Helium in their cores, but similarities end there. RR Lyrae stars pulsate, clump giants do not. RR Lyrae stars are metal-poor, clump giants are metal-rich. RR Lyrae stars are a part of an axisymmetric stellar halo, whereas clump giants form a 
bar. Stutz et al. (1999) suggested that the very red $(V-I)_{0}$ of the bulge RR Lyrae stars might have resulted from an unusual abundance of $\alpha$ elements. Why should a clump population which emerged in a different formation process share the same property?

The presence of the same type of color anomaly for different types of stars suggests that the effect might be unrelated to the physics of those stars. The investigated RR Lyrae and clump giants share two things in common. First, photometry of both types of stars comes from the OGLE, phase-I, project. Indeed, Paczyński et al. (1999) showed that OGLE-I $V$-magnitudes are $0.021 \mathrm{mag}$ fainter, and $I$-magnitudes $0.035 \mathrm{mag}$ brighter than better calibrated OGLE-II magnitudes. Therefore, the correct $(V-I)$ colors should be 0.056 bluer. Additionally, the new $(V-I)_{0}$ from the more homogeneous Baade's Window clump is bluer than Paczyński's \& Stanek's (1998) color, even when reduced to OGLE-I calibration. As a result, the $(V-I)_{0}$ anomaly shrinks and the remaining shift amounts to $\sim 0.11$ for both the RR Lyrae stars and clump giants. Second, Paczyński (1998) and Stutz et al. (1999) use the same extinction map (Stanek $1996)$ and the same coefficient of conversion from visual extinction $A_{V}$ to a color excess $E(V-I)$. The absolute values of the $A_{V} \mathrm{~s}$ are probably correct, because the zero-point of the extinction map was determined from the $(V-K)$ color, and $A_{V} / E(V-K)$ is very close to 1 . However, $R_{V I}=A_{V} / E(V-I)$ is not as secure, and has a pronounced effect on the obtained color.

The value and variation of $R_{V I}$ was thoroughly investigated by Woźniak \& Stanek (1996). The essence of the Woźniak \& Stanek (1996) method to determine differential extinction is an assumption that regions of the sky with a lower surface density of stars have higher extinction. This is quite a natural expectation, as far as the density of the underlying true population of stars does not depend on location. However, it is not obvious a priori how to convert a certain density of stars to an amount of visual extinction. Therefore, Woźniak \& Stanek (1996) used clump giants to calibrate their extinction. To make a calibration procedure completely unbiased would require, among other things, that the $V$-magnitudes of clump giants do not depend on their color [here ( $V-$ $\left.I)_{0}\right]$, that reddened and unreddened clump giants be drawn from the same parent population, and that clump giants were selected without any assumption about $R_{V I}$. None of those is true (for details see Popowski 2000).

Because the smaller selective extinction coefficient is not excluded by the current studies, I will assume $R_{V I}=2.1$ to match the $(V-I)_{0}$ colors of the bulge with the ones in the solar neighborhood. The color is a weak function of $[\mathrm{Fe} / \mathrm{H}]$, so this procedure is justified because the metallicities of the bulge and solar neighborhood are similar. The change in $R_{V I}$ from 2.5 to 2.1 will decrease the $I$-mag extinction by $0.11 \mathrm{mag}$, and increase the clump-based distance to the Galactic center by the same amount.

\section{Recalibration of Clump Giant Stars}

How do the bulge results bear on the distance to the LMC? The better photometry from Paczyński et al. (1999) and the modification of $R_{V I}$ influence the relative $\mathrm{RR}$ Lyrae and clump distances to the Galactic center. Thus, the $M_{I}(\mathrm{RC})$ $-[\mathrm{Fe} / \mathrm{H}]$ relation for clump giants used in the LMC, which was calibrated with 
respect to the baseline provided by $R R$ Lyrae stars (Udalski 1998a), changes. Assuming linearity of $M_{I}(\mathrm{RC})-[\mathrm{Fe} / \mathrm{H}]$ and making some small adjustments to $[\mathrm{Fe} / \mathrm{H}]$ used by Udalski (1998a), I find:

$$
M_{I}(\mathrm{RC})=-0.23+0.19[\mathrm{Fe} / \mathrm{H}],
$$

with a slope $0.10 \mathrm{mag} / \mathrm{dex}$ steeper than the original result. Such adjustment increases the best clump giant estimate from $\mu_{\mathrm{LMC}}=18.18 \pm 0.06$ to $\mu_{\mathrm{LMC}}=$ $18.27 \pm 0.07$. When distance estimates from the red clump, $R R$ Lyrae stars and eclipsing binary HV2274 are combined, one obtains $\mu_{\text {LMC }}=18.31 \pm 0.04$. The $1 \sigma$ uncertainty of this determination is only a formal error. The systematic errors are likely to dominate the true uncertainty. However, if the methods presented do not suffer from severe biases, then a distance to the LMC as long as 52-55 kpc ( $\left.\mu_{\text {LMC }} \approx 18.6-18.7\right)$ is highly disfavored by the current results.

\section{References}

Cole, A. A. 1998, ApJ, 500, L137

Feast, M. W. \& Catchpole, R. M. 1997, MNRAS, 286, L1

Girardi, L., Groenewegen, M. A. T., Weiss, A., \& Salaris, M. 1998, MNRAS, 301,149

Guinan, E. F., Fitzpatrick, E. L., Dewarf, L. E., et al. 1998, ApJ, 509, L21

Madore, B. F., Freedman, W. L., Silbermann, N., et al. 1999, ApJ, 515, 29

Nelson, C. A., Cook, K. H., Popowski, P., \& Alves, D. A. 2000, AJ, to appear in March 2000 issue [astro-ph/9910172]

Paczyński, B. 1998, Acta Astron., 48, 405

Paczyński, B. \& Stanek, K. Z. 1998, ApJ, 494, L219

Paczyński, B., Udalski, A., Szymański, M., Kubiak, M., Pietrzyński, G., Soszyński, I., Woźniak, P., \& Żebruń, K., 1999, Acta Astron., 49, 319

Popowski, P. 2000, ApJ, 528, L9

Popowski, P. \& Gould, A. 1999, in Post-Hipparcos Cosmic Candles, ed. A. Heck \& F. Caputo (Dordrecht: Kluwer), 53 [astro-ph/9808006]

Stanek, K. Z. 1996, ApJ, 460, L37

Stanek, K. Z., Zaritsky, D., \& Harris, J. 1998, ApJ, 500, L141

Stutz, A., Popowski, P., \& Gould, A. 1999, ApJ, 521, 206

Udalski, A. 1998a, Acta Astron., 48, 113

Udalski, A. 1998b, Acta Astron., 48, 383

Udalski, A., Pietrzyński, G., Woźniak, P., Szymański, M., Kubiak, M., \& Żebruń, K. 1998a, ApJ, 509, L25

Udalski, A., Szymański, M., Kubiak, M., Pietrzyński, G., Woźniak, P., \& Żebruń, K. 1998b, Acta Astron., 48, 1

Udalski, A., Szymański, M., Kubiak, M., Pietrzyński, G., Soszyński, L., Woźniak, P., \& Żebruń, K. 1999, Acta Astron., 49, 201 [astro-ph/9907236]

Walker, A. R. 1992, ApJ, 390, L81

Woźniak, P. \& Stanek, K. Z. 1996, ApJ, 464, 233 


\section{Discussion}

Darragh O'Donoghue: Your presentation is a little more than a party political propaganda talk. It will help now if you were to investigate why advocates of the long distance scale such as Feast \& Catchpole (1997) are wrong.

Piotr Popowski: It is enough if I prove that I am right, and not that they are wrong. We have three reliable methods which consistently cluster around a short distance to the LMC of about $45 \mathrm{kpc}$, and there is another potentially reliable method or standard candle (Cepheids) which gives a different result. I think that we should investigate what is going on with Cepheids (it is the best if this is done by (epheid people).

David Laney: The statistical arguments by Feast \& Catchpole have been demonstrated to be correct by Koen \& Laney and, using Monte Carlo simulations, by Pont. The error bar from the Monte Carlo simulations is closer to 0.15 than 0.1 , however.

Piotr Popowsti: This point has been raised by someone in the audience. No disagreement here, so I made no comment about it.

Giuseppe Bono: Two comments: 1. In a recent investigation by Romaniello et al. (1999) based on HST data of red clump stars, they found a distance modulus for LMC which seems to support the long distance scale. 2. Evolutionary models suggest that the RR Lyrae luminosity decreases with increasing metallicity.

Géza kovics: When you talk about distance of the LMC based on RR Lyrae stars you should also consider RRd stars. Applying them as distance indicators for the LMC yields $\approx 18.5$ mag for the distance modulus (Kovács \& Walker 1998). This is in agreement with the Cepheid B-W distance scale.

Piotr Popowski: The preliminary results of reanalysis of RRd stars from the MACHO group (David Alves and I are involved in this work) indicate that RRd stars' distance to the LMC is short as well. 